\title{
High-Resolution Keck Spectra of the Associated Absorption Lines in 3C 191
}

\author{
Frederick W. Hamann ${ }^{1}$, T.A. Barlow ${ }^{2}$, F.C. Chaffee ${ }^{3}$, C.B. Foltz ${ }^{4}$, R.J. Weymann ${ }^{5}$
}

\begin{abstract}
Associated absorption lines (AALs) are valuable probes of the gaseous environments near quasars. Here we discuss high-resolution $\left(6.7 \mathrm{~km} \mathrm{~s}^{-1}\right)$ spectra of the AALs in the radio-loud quasar 3C 191 (redshift $z=1.956$ ). The measured AALs have ionizations ranging from $\mathrm{Mg}$ I to $\mathrm{N} \mathrm{V}$, and multi-component profiles that are blueshifted by $\sim 400$ to $\sim 1400 \mathrm{~km} \mathrm{~s}^{-1}$ relative to the quasar's broad emission lines. These data yield the following new results.

1) The strengths of excited-state Si II* AALs indicate a density of $\sim 300 \mathrm{~cm}^{-3}$ in the $\mathrm{Si}^{+}$gas.

2) If the gas is photoionized, this density implies a distance of $\sim 28 \mathrm{kpc}$ from the quasar. Several arguments suggest that all of the lines form at approximately this distance.

3) The characteristic flow time from the quasar is thus $\sim 3 \times 10^{7} \mathrm{yr}$.

4) Strong Mg I AALs identify neutral gas with very low ionization parameter and high density. We estimate $n_{H} \gtrsim 5 \times 10^{4} \mathrm{~cm}^{-3}$ in this region, compared to $\sim 15 \mathrm{~cm}^{-3}$ where the $\mathrm{N} \mathrm{V}$ lines form.

5) The total column density is $N_{\mathrm{H}} \lesssim 4 \times 10^{18} \mathrm{~cm}^{-2}$ in the neutral gas and $N_{\mathrm{H}} \sim 2 \times 10^{20}$ $\mathrm{cm}^{-2}$ in the moderately ionized regions. These column densities are consistent with 3C 191's strong soft X-ray flux and the implied absence of soft X-ray absorption.

6) The total mass in the AAL outflow is $M \sim 2 \times 10^{9} \mathrm{M}_{\odot}$, assuming a global covering factor (as viewed from the quasar) of $\sim 10 \%$.

7) The absorbing gas only partially covers the background light source(s) along our line(s) of sight, requiring absorption in small clouds or filaments $<0.01 \mathrm{pc}$ across. The ratio $N_{H} / n_{H}$ implies that the clouds have radial (line-of-sight) thicknesses $\lesssim 0.2 \mathrm{pc}$.

These properties might characterize a sub-class of AALs that are physically related to quasars but form at large distances. We propose a model for the absorber in which pockets of dense neutral gas are surrounded by larger clouds of generally lower density and higher ionization. This outflowing material might be leftover from a blowout associated with a nuclear starburst, the onset of quasar activity or a past broad absorption line (BAL) wind phase.
\end{abstract}

Subject headings: Galaxies: active, Galaxies: starburst, Quasars: absorption lines, Quasars: general, Quasars: individual (3C 191)

\footnotetext{
${ }^{1}$ Department of Astronomy, University of Florida, 211 Bryant Space Science Center, Gainesville, FL 32611-2055, Internet: hamann@astro.ufl.edu

2Infrared Processing and Analysis Center, California Institute of Technology, MS 100-22, 770 South Wilson Ave., Pasadena, CA 91125

${ }^{3}$ California Association for Research in Astronomy, W.H. Keck Observatory, 65-1120 Mamalahoa Highway, Kamuela, HI 96734

${ }^{4}$ MMT Observatory, University of Arizona, 933 North Cherry Ave., Tucson, AZ 85721-0065

${ }^{5}$ Observatories of the Carnegie Institution of Washing-
}

\section{Introduction}

Associated absorption lines (AALs) are important diagnostics of the gaseous environments of quasars and active galactic nuclei (AGNs). The lines are defined empirically as having velocity widths less than a few hundred $\mathrm{km} \mathrm{s}^{-1}$ and absorption redshifts, $z_{a}$, within a few thousand $\mathrm{km} \mathrm{s}^{-1}$ of the quasar's emission-line redshift, $z_{e}$ (Weymann et al. 1979, Foltz et al. 1986). The

ton, 813 Santa Barbara Street, Pasadena, CA 91101-1292 
requirement for $z_{a} \approx z_{e}$ makes AALs more likely to be physically related to the quasars than are the other narrow absorption lines $\left(\right.$ at $z_{a} \ll z_{e}$ ) in quasar spectra (see Rauch 1998 for a review of the unrelated $z_{a} \ll z_{e}$ systems). The narrow velocity widths further distinguish AALs from the class of broad absorption lines (BALs), whose widths and maximum blueshifted velocities both typically exceed 10,000 $\mathrm{km} \mathrm{s}^{-1}$ (Weymann et al. 1991). BALs clearly form in high-velocity winds from quasar engines (e.g. Turnshek 1988), but AALs can form potentially in a variety of environments - ranging from energetic outflows like the BALs to relatively quiescent gas at large galactic or inter-galactic distances (see Hamann \& Brandt 2000 for a general review, also Tripp et al. 1996, Hamann et al. 1997a, Barlow \& Sargent 1997, Barlow, Hamann \& Sargent 1997). More work is needed to locate individual AAL absorbers, quantify their kinematic and physical properties, and understand the role of the AGNs and/or host galaxies in providing the source of their material and kinetic energy.

One interesting property is that, among radioloud quasars, AALs appear more frequently and with greater strength in sources with "steep" radio spectra and/or lobe-dominated radio morphologies (Wills et al. 1995, Barthel et al. 1997, Richards et al. 2000, also Brotherton et al. 1998 and references therein). This weak correlation is usually attributed to an orientation effect, whereby AAL regions reside preferentially near a disk or torus that is aligned perpendicular to the radio jet axis (but see Richards et al. 2000 for an alternative interpretation). However, it is not clear if this (possible) AAL geometry has its origins on small scales related to the inner black hole/accretion disk, or on much larger scales related to the host galaxy. It is also not clear if a disk-like geometry applies as well to the AALs in radio-quiet sources.

3C $191\left(\mathrm{Q} 0802+103, z_{e}=1.956\right)$ is a radioloud quasar having both strong AALs (Burbidge, Lynds \& Burbidge 1966, Stockton \& Lynds 1966, Williams et al. 1975, Anderson et al. 1987) and a bipolar, lobe-dominated radio structure (Akujor et al. 1994). It therefore follows the AALradio morphology correlation noted above. 3C 191 also provides a rare opportunity to define the distance between the quasar and the absorbing gas because several of its AALs arise from excited en- ergy states, e.g. C II* $\lambda 1336$ and Si II* $\lambda 1265,1533$ (Bahcall, Sargent \& Schmidt 1967, Williams et al. 1975). The strengths of the excited-state lines, compared to their resonant counterparts, C II $\lambda 1335$, Si II $\lambda 1260,1527$, provide measures of the gas density needed to populate the upper levels. The density in turn constrains the absorber's distance from the quasar, with the reasonable assumption that the gas is in photoioization equilibrium with the quasar radiation field.

Williams et al. (1975) already estimated a density of $n_{e} \sim 1000 \mathrm{~cm}^{-3}$ and a radial distance of $R \sim 10 \mathrm{kpc}$ for the AAL region in 3C 191. We reobserved 3C 191 with higher spectral resolution and wider wavelength coverage to 1 ) obtain more reliable densities as a function of velocity, 2) search for Fe II* AALs, which might be revealing of a much higher density environment (Wampler et al. 1995, Halpern et al. 1996), 3) revisit the question of this absorber's origin and location, and 4) obtain better constraints on the AAL region dynamics, abundances and overall physical structure.

Sections 2 and 3 below describe the observations and results. Section 4 provides measurements and analyses of the AALs. Section 5 draws further inferences and discusses physical models. Throughout this paper we define solar abundances by the meteoritic results in Grevesse \& Anders (1989), and we use atomic transition data from the compilation by Verner, Verner \& Ferland (1996).

\section{Observations Data Reductions}

We observed 3C 191 on 3 occasions using the High Resolution Echelle Spectrometer (HIRES, Vogt et al. 1994) on the Keck I telescope on Mauna Kea, Hawaii. On 13 December 1996 we obtained useful spectra from $\sim 3850 \AA$ to $\sim 5975 \AA$ in a total of $12,000 \mathrm{~s}$ exposure time. On 21 December 1997 we covered the wavelengths $\sim 6474$ to $\sim 8927$ $\AA$ in a total of $18,000 \mathrm{~s}$, and on 1 January 1998 we measured $\sim 3530 \AA$ to $\sim 4935 \AA$ in a total of 12,000 s. On each occasion, a $0.86^{\prime \prime}$ slit provided spectral resolution $\lambda / \Delta \lambda \approx 45,000$ or $6.7 \mathrm{~km} \mathrm{~s}^{-1}$, corresponding to 3 pixels on the $2048 \times 2048$ Tektronix CCD. Also on each occasion, we used two settings of the echelle grating and cross disperser to achieve continuous wavelength coverage across the quoted intervals. There is considerable overlap between 
instrument settings in the short wavelength spectra (December 1996 and January 1998), but almost none at longer wavelengths (December 1997). Wavelength regions not covered by both setups received half the total exposure times listed above.

We use standard techniques and a software package called $\mathrm{MAKEE}^{6}$ for the initial data reductions and spectral extractions. Flat-fielding and wavelength calibrations were achieved by measuring internal quartz and Th-Ar arc lamps, respectively, during each observing run. The wavelength solutions typically have root-mean-square uncertainties of $<0.22 \mathrm{~km} \mathrm{~s}^{-1}(<0.1$ CCD pixels).

We use the $\operatorname{IRAF}^{7}$ software package for additional data processing. In particular, we produce normalized spectra in each echelle order by fitting the continuum plus broad emission lines (BELs) with low-order polynomials. We then average together the overlapping echelle orders from all exposures on each night, weighting each pixel by the calculated noise (variance). There is no evidence for significant variability in the AALs measured in common on December 1996 and January 1998. We therefore combine these short-wavelength data into a single variance-weighted mean spectrum.

Sharp absorption lines corresponding to the telluric A and B bands of $\mathrm{O}_{2}$ overlap with the Fe II $\lambda 2587$ and $\lambda 2600$ AALs. We attempt to remove these telluric lines by dividing by the spectrum of a hot standard star measured the same night at the same airmass as 3C 191. Those efforts were only moderately successful (see Figure 2 below).

Finally, we calibrate the flux using lowresolution spectra of 3C 191 and a stellar standard obtained at the Lick Observatory on 2 December 1997. The Lick spectra were measured through an $8^{\prime \prime}$ slit under photometric conditions. The final fluxes should have $3 \sigma$ uncertainties of $\lesssim 7 \%$.

\section{Results and Measurements}

Figure 1 shows part of the combined HIRES spectrum, with pixels binned to match (roughly)

\footnotetext{
${ }^{6}$ MAKEE was developed by T.A. Barlow specifically for reduction of Keck HIRES data. It is freely available on the world wide web at the Keck Observatory home page, http://www2.keck.hawaii.edu:3636/

${ }^{7}$ IRAF is maintained and distributed by the National Optical Astronomy Observatories, in cooperation with the National Science Foundation.
}

the plot resolution. Figure 2 shows velocity profiles for the strongest AALs. The quasar rest frame is defined here and throughout this paper by the emission-line redshift, $z_{e}=1.956$, as measured by Tytler \& Fan (1992) from C IV $\lambda 1549$, He II $\lambda 1640, \mathrm{O}$ III] $\lambda 1664$, and C III] $\lambda 1909$. To improve the signal-to-noise ratio across the $\mathrm{N} \mathrm{V}$ AALs, Figure 2 plots the linear average of the $\mathrm{N}$ V $\lambda \lambda 1239,1243$ doublet pair (labeled "N V average" in the figure).

Table 1 lists all of the detected AALs with their rest-frame equivalent widths $\left(W_{\lambda}\right)$ and corresponding minimum column densities $(\log N)$. To accommodate some of the line profile complexity, we measure these quantities in three velocity intervals: $-1400 \leq \Delta v_{1}<-1160 \mathrm{~km} \mathrm{~s}^{-1}$, $-1160 \leq \Delta v_{2}<-810 \mathrm{~km} \mathrm{~s}^{-1},-810 \leq \Delta v_{3} \leq$ $-400 \mathrm{~km} \mathrm{~s}^{-1}$, plus the total profiles from -1400 to $-400 \mathrm{~km} \mathrm{~s}^{-1}$. The equivalent widths listed for "N V average" are based on the average profile in this doublet. The total equivalent width given for $\mathrm{C} \mathrm{II+C} \mathrm{II*} \mathrm{applies} \mathrm{to} \mathrm{the} \mathrm{entire} \mathrm{blend} \mathrm{of}$ $\lambda 1335+\lambda 1336$ (Fig. 2). For C IV $\lambda 1548$ and $\lambda 1551$, some values of $W_{\lambda}$ are omitted because those velocity intervals are dominated by the other component of this blended doublet. The middle interval $\left(v_{2}\right)$ has equal contributions from both C IV components. The total C IV equivalent width (listed separately as "C IV total" in the table) applies to the entire blend. The equivalent widths for Fe II $\lambda 2600$ and Fe II $\lambda 2587$ are based on crude linear interpolations across the residual narrow $\mathrm{O}_{2}$ absorption lines in these profiles (see Fig. 2 and $\S 2$ ).

The uncertainties in the $W_{\lambda}$ results are dominated by the (largely) subjective continuum placement. Repeated measurements with different continuum levels suggest that the $1 \sigma$ uncertainties are $\sim 5 \%$ for lines with $W_{\lambda} \gtrsim 3 \AA, \sim 10 \%$ for $0.3 \lesssim W_{\lambda} \lesssim 3 \AA$, and $\sim 20 \%$ for weaker lines. A few weak or blended lines are marked with '?' in Table 1, indicating uncertainties $>20 \%$.

The column densities listed in Table 1 are discussed in $\S 4.3$ below.

\section{Analysis of the AAL System}

\subsection{Line Profiles and Kinematics}

Most of the AALs have complex, multicomponent velocity profiles. These components tend to be broader in the stronger (deeper) lines. 
For example, the full width at half minimum (FWHM) of the feature at $-542 \mathrm{~km} \mathrm{~s}^{-1}$ increases from $\sim 13-15 \mathrm{~km} \mathrm{~s}^{-1}$ in the Si II, Al II and Al III lines, to $\sim 30 \mathrm{~km} \mathrm{~s}^{-1}$ in $\mathrm{Mg}$ II, to $>100 \mathrm{~km} \mathrm{~s}^{-1}$ in C IV. Other components, e.g. near $-1000 \mathrm{~km} \mathrm{~s}^{-1}$, blend together and lose their distinct identities altogether in the stronger lines. One of the strongest lines, Ly $\alpha$, has no discrete components at all only a single broad trough with $\mathrm{FWHM} \approx 920$ $\mathrm{km} \mathrm{s}^{-1}$.

Overall, the AALs span blueshifted velocities from $\sim 400$ to $\sim 1400 \mathrm{~km} \mathrm{~s}^{-1}$ (relative to $z_{e}=$ 1.956). The absolute velocities in the quasar rest frame are uncertain by up to a few hundred $\mathrm{km} \mathrm{s}^{-1}$ because the BELs, used to define $z_{e}(\S 3)$, can themselves be either blue or redshifted relative to the quasar (see Tytler \& Fan 1992, Marziani et al. 1996). Nonetheless, the measured velocity shifts give strong evidence for an outflow from the quasar.

\subsection{Partial Line-of-Sight Coverage}

Some of the strongest AALs, such as Ly $\alpha$ and the C IV doublet, have flat-bottomed profiles that do not reach zero intensity. This profile shape suggests that the transitions are very optically thick. In principle, the non-zero line intensities could also result from many narrow, unresolved line components that only partially overlap in velocity. However, such line components cannot be narrower than a thermal line width. In an ionized plasma with a nominal temperature of $\sim 10^{4} \mathrm{~K}$, the thermal speeds of $\mathrm{H}$ and $\mathrm{C}$ correspond to FWHMs of $\sim 21$ and $\sim 6.5 \mathrm{~km} \mathrm{~s}^{-1}$, respectively. These minimum line widths are greater than or equal to the resolution of our spectra. Therefore, the lines are fully resolved and the flat-bottomed profiles must be caused by optically thick absorption plus unabsorbed flux that fills in the bottoms of the troughs. This filling-in can occur if the absorber does not fully cover the background light source(s) along our line(s) of sight (see Hamann \& Brandt 2000). In general, fully resolved line intensities depend on the line-of-sight coverage fraction, $C_{f}$, as,

$$
I_{v}=\left(1-C_{f}\right) I_{o}+C_{f} I_{o} e^{-\tau_{v}}
$$

where $0 \leq C_{f} \leq 1, I_{o}$ is the unabsorbed intensity at a given profile velocity $v$, and $I_{v}$ and $\tau_{v}$ are the measured intensity and true optical depth at that velocity. For $\tau_{v} \gg 1$, the coverage fraction simply equals the depth of the line below the continuum, such that,

$$
C_{f} \geq 1-\frac{I_{v}}{I_{o}}
$$

If the flat-bottom lines in 3C 191 are, in fact, optically thick, the residual intensities in their cores imply $C_{f} \approx 96 \%$ for the C IV doublet, $\sim 93 \%$ for Si IV, and $\sim 88 \%$ for Ly $\alpha$.

Further evidence for $C_{f}<1$ appears in Figure 3 , which compares the measured profiles in $\mathrm{Mg}$ II $\lambda 2804$, Al III $\lambda 1863$ and Fe II $\lambda 2344$ to predictions based on their stronger multiplet counterparts $\mathrm{Mg}$ II $\lambda 2796, \mathrm{Al}$ III $\lambda 1855$ and Fe II $\lambda 2383$. The predictions follow by 1 ) deriving the apparent optical depths, $\tau_{v}^{a} \equiv-\ln \left(I_{v} / I_{o}\right)$, in the stronger lines (i.e. assuming $C_{f}=1$ in Eqn. 1), 2) calculating $\tau_{v}^{a}$ in the weaker transitions by scaling by the relative $g f \lambda$ values, and 3) using those calculated optical depths to predict $I_{v}$ in the weaker lines (see also Barlow \& Sargent 1997).

The weaker predicted compared to observed lines in Figure 3 require $C_{f}<1$. The average intensities from -1025 to $-860 \mathrm{~km} \mathrm{~s}^{-1}$ (near the line centers) differ between the observed and predicted profiles at significance levels of $\sim 16 \sigma$ in the $\mathrm{Mg}$ II doublet, $\sim 14 \sigma$ in $\mathrm{Al}$ III, and $\sim 2.7 \sigma$ in the Fe II pair (where $\sigma$ represents 1 standard deviation in the photon counting statistics). The average coverage fractions in this velocity interval are $\left\langle C_{f}\right\rangle=0.84 \pm 0.01$ in $\mathrm{Mg}$ II, $0.60 \pm 0.02$ in Al III, and $0.57+0.13 /-0.09$ in Fe II (see Hamann et al. 1997a, Barlow \& Sargent 1997, Petitjean \& Srianand 1999 for explicit equations). In the deepest part of the lines, the coverage fractions are larger. For example, notice that the core of the Al III $\lambda 1855$ line dips well below $1-\left\langle C_{f}\right\rangle \approx 0.4$ in Figure 2 . The line intensities in the narrower interval -950 to $-915 \mathrm{~km} \mathrm{~s}^{-1}$ imply $\left\langle C_{f}\right\rangle=0.89 \pm 0.02$ in $\mathrm{Mg}$ II, $0.79 \pm 0.02$ in Al III, and $0.68+0.22 /-0.9$ in Fe II. Similar variations in $C_{f}$ across AAL profiles have been noted previously in other quasars (Barlow et al. 1997).

\subsection{Column Densities}

The column densities in Table 1 are lower limits derived from the apparent line optical depths as follows,

$$
N \geq \frac{m_{e} c}{\pi e^{2} f \lambda_{o}} \int \tau_{v}^{a} d v
$$


where $\lambda_{o}$ is the rest wavelength of the line (see Savage \& Sembach 1991). The equality in Equation 3 holds only if $C_{f}=1$ and the line absorption is fully resolved. Otherwise the result is a lower limit. To avoid spurious spikes in the $\tau_{v}^{a}$ distributions (e.g. where $I_{v} \lesssim 0$ in "noisy" lines), we first smooth the spectra several times with a binomial algorithm and then impose an arbitrary upper limit of $\tau_{v}^{a} \leq 6$ at each data point. Tests show that the integrated results are not sensitive to the value of this upper limit, as long as it is not much above $\sim 10$. Table 1 lists the resulting minimum column densities in each of the velocity intervals $\Delta v_{1}, \Delta v_{2}$ and $\Delta v_{3}$, and in the total profiles (see $\S 3)$.

The total column density listed for $\mathrm{C} \mathrm{II}+\mathrm{C} \mathrm{II}^{*}$ comes from treating this blended pair as a single line with $1 / 2$ the average $\mathrm{C}$ II $\lambda 1335+\mathrm{C} \mathrm{II}^{*} \lambda 1336$ gf value. The column density given as "C IV total" is the sum of contributions from $\Delta v_{1}$ in $\lambda 1548$ plus $\Delta v_{2}$ and $\Delta v_{3}$ in $\lambda 1551$. This estimate should approximate the total C IV column density. Finally, the results for "N V average" are derived from the average profile of this doublet (Fig. 2, $\S 3$ ) assuming an average $f$ value.

The evidence for partial coverage in $\S 4.2$ implies that the column densities listed in Table 1 are, in fact, just lower limits. These limits could be orders of magnitude too low for strong, flatbottomed lines like Ly $\alpha$, Si III $\lambda 1206$, Si IV $\lambda \lambda 1394,1403$ and C IV $\lambda \lambda 1548,1551$ (see Figure 2). For weak and intermediate lines, we can estimate the true column densities by adopting nominal covering fractions in our derivations of the $\tau_{v}^{a}$ (using Equation 1). We adopt $C_{f}=0.85$ for the $\mathrm{Mg}$ II doublet and $C_{f}=0.7$ for the other lines (§4.2). The resulting corrected column densities are listed for the total profiles under the heading "Tot $\left(C_{f}<1\right) "$ in Table 1.

\subsection{Ionization}

The metallic AALs range in ionization from $\mathrm{Mg}$ I $\lambda 2853$ to $\mathrm{N} \mathrm{V} \lambda 1239,1243$. This range of ions requires a range of distinct ionization zones. In particular, $\mathrm{Mg}$ I indicates a neutral gas component that cannot survive in the highly-ionized $\mathrm{N} \mathrm{V}$ region. The upper limit to the ionization is unknown because important lines of higher ionization species, such as O VI $\lambda \lambda 1032,1038$, are outside our wavelength coverage. The degree of neutrality in the $\mathrm{Mg}$ I zone can be inferred from theoretical models.

We examine theoretical clouds in photoionization equilibrium with the quasar radiation field using the computer code CLOUDY (version 90.05, Ferland et al. 1998). The ionizing spectrum has the form $L_{\nu} \propto \nu^{\alpha}$, where $\alpha=-1.6$ for $\nu \geq \nu_{L L}$ and $\alpha=-0.7$ for $\nu<\nu_{L L}$ (where $\nu_{L L}$ is the frequency at the H I Lyman limit). The spectral slope at $\nu<\nu_{L L}$ approximates the measured restframe UV spectrum from $\sim 1200 \AA$ to $\sim 2300 \AA$ (Fig. 1). The slope in the Lyman continuum is not known, but a spectral steepening to $\alpha \sim-1.6$ at $\nu \gtrsim \nu_{L L}$ is consistent with the best observations of other luminous quasars (Hamann, Netzer \& Shields 2000, Zheng et al. 1997, Laor et al. 1997). The ionization parameter, $U$, is defined as the dimensionless ratio of hydrogen particle to hydrogen-ionizing photon densities at the illuminated face of the clouds,

$$
U \equiv \frac{1}{4 \pi c R^{2} n_{H}} \int_{\nu_{L L}}^{\infty} \frac{L_{\nu}}{h \nu} d \nu
$$

where $L_{\nu}$ is the luminosity density of the quasar spectrum, $R$ is the distance between the absorber and the quasar, and $n_{H}$ is the total hydrogen density (in $\mathrm{H} \mathrm{I}+\mathrm{H}$ II).

A critical question is whether or not the absorber contains an $\mathrm{H}$ II-H I recombination front. If there is such a front, the absorption of Lyman continuum photons might allow low-ionization species like Si II, Al II and Fe II to exist physically adjacent to the high-ionization $\mathrm{C}$ IV and $\mathrm{N}$ V region, i.e. at essentially the same $R$. Individual clouds (at a given $R$ and $n_{H}$ ) could have high $U$ at their illuminated surface and still host low-ionization species behind the front. If, on the other hand, there is no ionization front, the absorber would be optically thin in the Lyman continuum $\left(\tau_{L C} \lesssim 1\right)$ and there could be no (significant) gradient in the ionization level due to the absorber's own opacity. The observed range of ionizations would then require a variety of clouds having different $U$ values and therefore very different $n_{H}$ and/or $R$ (Eqn. 4).

A key constraint comes from Mg I (Arav et al. 2000). Its ionization potential, $7.65 \mathrm{eV}$ (corresponding to $\lambda \sim 1620 \AA$ ), is well below the H I Lyman limit of $13.6 \mathrm{eV}(912 \AA)$. Therefore $\mathrm{Mg}$ I cannot be shielded by the presence of an H II- 
H I recombination front. Explicit CLOUDY calculations show that clouds with $U$ high enough to amply support N V near their illuminated surface cannot also support significant amounts of $\mathrm{Mg} \mathrm{I}$ behind an $\mathrm{H} \mathrm{II-H} \mathrm{I} \mathrm{front.} \mathrm{Essentially} \mathrm{all} \mathrm{of} \mathrm{the}$ magnesium behind the front is in the form of $\mathrm{Mg}$ II because the strong photon flux at $912 \lesssim \lambda \lesssim 1620$ $\AA$ photoionizes $\mathrm{Mg}$ I, while the absence of photons at $\lambda \lesssim 912 \AA$ prevents the formation of Mg III. (Al, Si and Fe behave similarly.) The observed ratio of Mg I/Mg II column densities, $\sim 0.05$ (Table 1 ), is several orders of magnitude larger than the predicted value everywhere in these model clouds. Further calculations with $\log U=-2$ to -3 similarly underpredict $N(\mathrm{MgI}) / N(\mathrm{MgII})$ by factors of 10-20 compared to Table 1.

The only way to match the observed $N(\mathrm{MgI}) / N(\mathrm{MgII})$ ratio in high- $U$ clouds is to invoke an additional opacity source to suppress the flux between 912 and $1620 \AA$ by a factor of at least ten. Bound-free absorption by neutral metals cannot provide this opacity because the column densities implied by the neutral lines are too low (e.g. $\left.\log N(\mathrm{Mg} \mathrm{I})\left(\mathrm{cm}^{-2}\right) \approx 13.2\right)$; moreover, we do not observe absorption edges at the appropriate wavelengths (e.g. at $1620 \AA$ for $\mathrm{Mg} \mathrm{I}$ ). Molecules, such as $\mathrm{H}_{2}$, also cannot be important opacity sources because there are no edges at their dissociation energies (e.g. at $4.48 \mathrm{eV}$ for $\mathrm{H}_{2}$ ). The only viable possibility is dust inside the absorbing region. However, the required large reduction in UV flux should produce significant reddening or perhaps a broad $\sim 2200 \AA$ absorption feature (e.g. Cardelli, Clayton \& Mathis 1989, Fall \& Pei 1989, Brotherton et al. 1998, Meurer, Heckman \& Calzetti 1999 and references therein). There is no evidence for these dust signatures in 3C 191 (compare Fig. 1 and the UV slope estimated above with O'Brien, Gondhalekar \& Wilson 1988 and Zheng et al. 1997).

The large observed $N(\mathrm{MgI}) / N(\mathrm{MgII})$ ratio therefore cannot be attributed to radiative shielding; rather, it requires neutral gas with very low ionization parameter. $\mathrm{Mg} \mathrm{I}$ and $\mathrm{N} \mathrm{V}$ together imply that the overall absorber contains a wide range of $U$ values and therefore a wide range in $n_{H}$ and/or $R$.

Figure 4 shows theoretical ionization fractions for clouds with $\tau_{L C} \lesssim 1$ and different $U$ (see Hamann 1997 for more details). Results for $\log U \lesssim-5$ are not shown because they are increasingly sensitive to uncertain factors, such as the input volume density, the derived temperature, and the details of molecule formation. Nonetheless, it seems clear that the measured ratio of Mg I/Mg II column densities requires predominantly neutral gas with $\log U \lesssim-5$. In contrast, the strong $\mathrm{N} V$ AALs identify a highly-ionized region where $\log U \sim-1.5$ is more representative. Intermediate ionizations are also present; for example, the ratio of $\mathrm{Al} \mathrm{II/Al} \mathrm{III} \mathrm{column} \mathrm{densities}$ indicates $\log U \approx-2.8$ in Figure 4 .

Overall there is a trend for increasing column densities in higher ionization species. In particular, the high-ionization lines are all saturated (Figure $2, \S 4.3$ ), and the inferred ratios of Si II/Si III, $\mathrm{Al}$ II/Al III and C II/C IV column densities are all above unity (Table 1). Clearly, there is much more highly-ionized compared to neutral gas in the overall AAL region.

\subsection{Total Column Density and X-ray Ab- sorption}

We can quantify these statements by estimating the total column density, $N_{H}$ (in $\mathrm{H} \mathrm{I}+\mathrm{H}$ II), in different regions. For example, an upper limit on $N_{H} \approx N(\mathrm{H} \mathrm{I})$ in the neutral gas follows from the measured column density in $\mathrm{Mg}$ I. If the $\mathrm{Mg} / \mathrm{H}$ abundance ratio is roughly solar, i.e. $\log (\mathrm{Mg} / \mathrm{H}) \approx$ -4.4 (which would be consistent with AALs in other quasars - Petitjean, Rauch \& Carswell 1994, Hamann 1997, Hamann \& Ferland 1999), and the $\mathrm{Mg}$ I ionization fraction is conservatively $\log f(\mathrm{MgI}) \gtrsim-1$ in the neutral gas, then $N_{\mathrm{H}} \approx$ $N(\mathrm{H}$ I $)$ should be $\lesssim 5.4$ dex larger than $N(\mathrm{Mg}$ I $)$ in Table 1. Therefore, we expect $\log N_{\mathrm{H}}\left(\mathrm{cm}^{-2}\right) \lesssim$ 18.6 in the neutral absorber.

For the higher ions, we assume the ionization fractions are near their peaks in Figure 4 to derive conservatively low estimates of $N_{\mathrm{H}}$ in their line-forming regions. Again assuming solar metal-to-hydrogen abundance ratios, we find $\log N_{\mathrm{H}}\left(\mathrm{cm}^{-2}\right) \approx 19.5$ and 19.4 based on the measured Si II and Al II column densities, respectively, $\log N_{\mathrm{H}}\left(\mathrm{cm}^{-2}\right) \approx 20.3$ based on $\mathrm{Al}$ III, and $>19.6$ based on a minimum Si III+Si IV column density. The total column density in more highly ionized regions could be even larger, given the saturated absorption in Si IV, C IV and N V (§3). In any case, $N_{\mathrm{H}}$ is at least 1.7 dex larger at moderate to 
high ionizations compared to the neutral gas.

The total column density of $\log N_{\mathrm{H}}\left(\mathrm{cm}^{-2}\right) \approx$ 20.3 derived above corresponds to a continuum optical depth of $\lesssim 5 \%$ at $1 \mathrm{keV}$ (with the maximum value obtaining for a neutral absorber, Morrison \& McCammon 1983). This prediction based on the AALs is consistent with the observed normal ratio of soft X-ray to UV continuum fluxes in 3C 191 (Wilkes et al. 1994). There is evidently no significant soft X-ray absorption in this source. This situation contrasts with the extreme X-ray absorption in BAL quasars, and with the strong to moderate X-ray absorption found in other AGNs with significant AALs (Green \& Mathur 1996, Gallagher et al. 1999, Crenshaw et al. 1999, Brandt, Laor \& Wills 2000).

\subsection{Excited-State AALs: Space Density and Radial Distance}

Several excited-state AALs of C $\mathrm{II}^{*}$ and Si II* are present (Table 1 and Figure 2). These features arise from ground ${ }^{2} P^{o}$ multiplets that should behave approximately as 2-level atoms - with level populations controlled by collisional processes and forbidden radiative decays (see Morris et al. 1986). Bahcall \& Wolf (1968) showed that electron impacts are more important than proton collisions for temperatures below $\sim 20,000 \mathrm{~K}$, which should be appropriate for the $\mathrm{C}^{+}$and $\mathrm{Si}^{+}$regions in photoionized plasmas. Absorption lines from the excited states, e.g. Si $\mathrm{II}^{*} \lambda 1533$ and $\mathrm{C} \mathrm{II}^{*} \lambda 1336$, compared to the resonance transitions, Si II $\lambda 1527$ and C II $\lambda 1335$, provide direct measures of the level populations and thus the electron density. The density is given by,

$$
n_{e} \approx n_{c r}\left(\frac{2 N_{l o}}{N_{u p}}-1\right)^{-1}
$$

where $N_{l o}$ and $N_{u p}$ are the column densities measured from the ground and excited-state lines, respectively, and $n_{c r}$ is the critical electron density of the upper state (at which collisional deexcitation equals radiative decays). Note that $N_{l o} / N_{u p}$ must be $\geq 0.5$. The critical density scales with the electron temperature roughly as $T^{1 / 2}$. For $T=8000 \mathrm{~K}$, we derive $n_{c r} \approx 34 \mathrm{~cm}^{-3}$ for the $\mathrm{C}^{+}$upper state and $n_{c r} \approx 1580 \mathrm{~cm}^{-3}$ for $\mathrm{Si}^{+}$ (using collision strengths from Osterbrock 1989 and radiative decay rates from Mendoza 1983 and Galavis, Mendoza \& Zeippen 1998).
Inspection of the similar Si II $\lambda 1527$ and Si II* $\lambda 1533$ profiles indicates a similar density at all velocities. In the main AAL components, between -1160 and $-810 \mathrm{~km} \mathrm{~s}^{-1}\left(\Delta v_{2}\right.$ in Table 1$)$, the column densities inferred from these lines imply $n_{e} \approx 510 \mathrm{~cm}^{-3}$ if $C_{f}=1$ or $n_{e} \approx 300 \mathrm{~cm}^{-3}$ if $C_{f} \approx 0.7$. The narrow absorption component at $-542 \mathrm{~km} \mathrm{~s}^{-1}$ in these transitions similarly indicates $n_{e} \approx 395 \mathrm{~cm}^{-3}$ if $C_{f} \approx 1$ or $n_{e} \approx 300$ $\mathrm{cm}^{-3}$ if $C_{f}=0.7$.

The measured C II $\lambda 1335$ and $\mathrm{C}$ II* $\lambda 1336$ lines are noisy and may be saturated. Nonetheless, their relative strengths suggest densities above the critical value of $\sim 34 \mathrm{~cm}^{-3}-$ consistent with the $\mathrm{Si} \mathrm{II} / \mathrm{Si} \mathrm{II*}$ results. We do not detect any Fe II* lines, even though several strong transitions lie within our spectral coverage. An explicit calculation (kindly performed by G. Ferland using CLOUDY) shows that for $n_{e} \approx 300 \mathrm{~cm}^{-3}$ and $T \approx 8000 \mathrm{~K}$ the strongest measurable $\mathrm{Fe} \mathrm{II*}$ line, $\lambda 2349$, should be $\gtrsim 40$ times weaker than $\mathrm{Fe}$ II $\lambda 2383$ and therefore below our detection threshold. We therefore adopt $n_{e} \approx 300 \mathrm{~cm}^{-3}$ as being representative of the low-ionization gas.

Assuming the gas is photoionized, we can combine this density with $U$ from $\S 4.4$ to estimate the distance, $R$, between the absorber and the quasar (Eqn. 4). We approximate $\mathrm{E}_{\nu}$ in the Lyman continuum by a power law of the form,

$$
L_{\nu}=L_{L L}\left(\frac{\nu}{\nu_{L L}}\right)^{\alpha}
$$

where $L_{L L}$ is the luminosity density at the Lyman limit. Equation 4 then yields,

$$
R=\left(\frac{-L_{L L}}{4 \pi c h n_{H} U \alpha}\right)^{1 / 2}
$$

for $\alpha<0$. We estimate $L_{L L} \approx 3.6 \times 10^{31} \mathrm{ergs} \mathrm{s}^{-1}$ $\mathrm{Hz}^{-1}$ in the quasar rest frame by extrapolation from the fluxes in Figure 1 (assuming a cosmology with $H_{o}=65 \mathrm{~km} \mathrm{~s}^{-1} \mathrm{Mpc}^{-1}$ and $q_{o}=\Omega_{M} / 2-$ $\Omega_{\Lambda}=0$; Carroll, Press \& Turner 1992). We again adopt $\alpha=-1.6$ in the Lyman continuum (§4.4).

If we choose $n_{\mathrm{H}} \approx n_{e} \approx 300 \mathrm{~cm}^{-3}$ and a nominal value of $\log U \approx-2.8$ in the Si II zone (based on the $\mathrm{Al}$ II/Al III column densities, §4.4), the radial distance of that zone should be,

$$
R \approx 28 \mathrm{kpc}
$$


This result is not sensitive to the assumed spectral shape because the photon energies that control the Si II ionization are also most important in the definition of $U$ (i.e. near the $\mathrm{H}$ I Lyman limit, Eqn. 4). The value of $U$ presents a much larger uncertainty. Williams et al. (1975) derived a smaller distance of $R \approx 10 \mathrm{kpc}$ partly because they combined a density based on $\mathrm{Si}$ II/Si II* with a higher $U$ value based on Si IV, C IV and N V. That derivation, therefore, implicitly assumed that the Si II gas is radiatively shielded downstream from the $\mathrm{C}$ IV, etc. region. We have assumed that shielding is not important in the Si II zone, based on the Mg I strength ( $\$ 4.4$ and $\S 5.1$ item 3 ).

\subsection{Abundance Ratios}

We cannot derive the metallicity of the AAL gas because the only measured $\mathrm{H}$ line, $\mathrm{Ly} \alpha$, is severely saturated. The only possibility is to use some of the weaker lines, e.g. of $\mathrm{Mg}, \mathrm{Al}, \mathrm{Si}$ and $\mathrm{Fe}$, to estimate the relative abundance ratios in these elements. The logarithmic abundance ratio of any two elements $a$ and $b$ can be written,

$$
\left[\frac{a}{b}\right]=\log \left(\frac{N\left(a_{i}\right)}{N\left(b_{j}\right)}\right)+\log \left(\frac{f\left(b_{j}\right)}{f\left(a_{i}\right)}\right)+\log \left(\frac{b}{a}\right)_{\odot}
$$

where $(b / a)_{\odot}$ is the solar abundance ratio, and $N$ and $f$ are respectively the column densities and ionization fractions of element $a$ in ion state $i$, etc.

Figure 5 shows theoretical values of the normalized ionization corrections, defined here as $I C \equiv$ $\log \left(f\left(b_{j}\right) / f\left(a_{i}\right)\right)+\log (b / a)_{\odot}$, based on the calculations in $\S 4.4$ (Figure 4 ). Figure 5 shows that $I C$ for $\mathrm{Mg}$ II/Fe II and Si II/Fe II have minimum values near zero (in the log), which provide firm lower limits on the $\mathrm{Fe} / \mathrm{Si}$ and $\mathrm{Fe} / \mathrm{Mg}$ abundances (see Hamann 1997 and Hamann \& Ferland 1999 for more discussion). With column densities from Table 1, the corresponding abundance limits are $[\mathrm{Fe} / \mathrm{Mg}] \gtrsim-0.3$ and $[\mathrm{Fe} / \mathrm{Si}] \gtrsim-0.9$. The lowest ratios would obtain only if $-5.2 \lesssim \log U \lesssim-4.5$, but that would imply $\mathrm{Al} / \mathrm{Mg}$ and $\mathrm{Al} / \mathrm{Si}$ abundances several times above solar (Table 1 and Fig. 5 ). If the actual $U$ values are instead in the range $-3.4 \lesssim \log U \lesssim-2.8$ (consistent with our estimate of $\log U \sim-2.8$ based on Al II/Al III, §4.4), then we would derive roughly solar ratios among all of these elements.

Given the uncertainties in $U$, and the fact that different line forming regions can have different $U$ values (§4.4), we conclude only that the relative metal abundances are broadly consistent with solar, with uncertainties of factors of several.

\section{Discussion}

\subsection{Implications for the Structure and Dynamics}

The results in $\S 3$ and $\S 4$ lead to following important conclusions regarding the AAL environment.

1) We have already noted that the absorber has a complex velocity structure that appears qualitatively similar in all lines and ions (§4.1). Stronger AALs, e.g. of higher ionization, are more smoothly distributed in velocity, but it seems clear that all of the lines trace the same overall physical structure. We conclude that the radial distance $R \approx 28 \mathrm{kpc}$ derived for the $\mathrm{Si}^{+}$zone (\$4.6) should be roughly characteristic of the entire AAL region (see also item 4 below).

2) The AAL gas appears to be outflowing from the quasar at velocities from $\sim 400$ to $\sim 1400$ $\mathrm{km} \mathrm{s}^{-1}$ (§4.1). If the gas is not accelerating (or decelerating), then the time scale for this outflow reaching radius $R$ is

$$
t \approx 3 \times 10^{7}\left(\frac{R}{28 \mathrm{kpc}}\right)\left(\frac{1000 \mathrm{~km} \mathrm{~s}^{-1}}{v}\right) \mathrm{yr}
$$

where $v$ is a characteristic velocity. The overall AAL region will acquire a radial thickness during this expansion because different gas components move at different speeds. In 3C 191, the radial thickness, $\Delta R$, could be comparable to the radius, $R \approx 28 \mathrm{kpc}$, because the velocity dispersion implied by the line widths, $\Delta v \approx 1000 \mathrm{~km} \mathrm{~s}^{-1}$, is similar to the average flow speed.

3) The significant presence of $\mathrm{Mg} \mathrm{I}$, compared to Mg II, identifies a neutral gas component whose survival cannot be attributed to radiative shielding downstream from an $\mathrm{H}$ II-H I recombination front (§4.4). The Mg I region must have a low ionization parameter and therefore a high density. If we adopt $n_{H} \approx 300 \mathrm{~cm}^{-3}$ and $\log U \approx-2.8$ for the Si II region ( $\$ 4.6)$, then simple scaling based on $\log U \lesssim-5$ in the neutral gas (§4.4) implies $n_{H} \gtrsim 5 \times 10^{4} \mathrm{~cm}^{-3}$ in that region. In contrast, $\log U \sim-1.5$ in the $\mathrm{N} \mathrm{V}$ zone indicates $n_{H} \sim 15$ $\mathrm{cm}^{-3}$ there. 
4) The Si II $\lambda 1527$ and Si II* $\lambda 1533$ line profiles are surprisingly similar (Fig. 2) given the wide range of densities present. The nearly constant line ratio indicates a nearly constant density (within a factor of $\sim 2$ ) at all velocities. This result could be caused by a selection bias: the absorber may have a wide range of densities at each velocity, but the specific ionization requirements of $\mathrm{Si}^{+}$might persistently lead to just a narrow range in $U$ and therefore $n_{\mathrm{H}}$ controlling the Si II and Si II* line strengths. In any case, the similar $n_{\mathrm{H}}$ values inferred for different Si II velocity components support the argument (item 1 above) that the AAL region does not span a wide range in radial distance from the quasar.

5) Partial line-of-sight coverage of the quasar emission sources (§4.2) implies that the AAL clouds have characteristic sizes similar to or smaller than the projected area of the emitters. The Ly $\alpha$ and C IV absorption lines sit atop strong BELs (Fig. 2); they might fully cover the continuum source while partly covering the larger BEL region. The characteristic size of these absorbers might therefore be as large as $0.1-1$ pc (i.e. the size of the BEL region, e.g. Peterson 1993, Kaspi et al. 2000). However, the partial coverage inferred from the $\mathrm{Mg}$ II, Al III and Fe II lines must involve the continuum source, which requires absorber size scales $<0.01 \mathrm{pc}$ (for standard accretion disk models of the continuum emission, Netzer 1992).

6) Another constraint on the size scales comes from the ratio $N_{H} / n_{H}$. If the absorbing gas completely fills the volume it encompasses, then the radial thickness of the entire absorber would be of order,

$$
\Delta R \approx 0.2\left(\frac{N_{H}}{2 \times 10^{20} \mathrm{~cm}^{-2}}\right)\left(\frac{300 \mathrm{~cm}^{-3}}{n_{H}}\right) \mathrm{pc}
$$

using parameters from $\S 4.5$ and $\S 4.6$. However, if the absorber is composed of discrete clouds that fill only part of the encompassed volume, then the overall AAL region could have a much greater radial thickness (item 2 above) while the individuals clouds are small compared to $\Delta R$ in Equation 11.

7) The mass of the AAL region, $M$, depends on its radial distance, total column density and global covering factor, $Q \equiv \Omega / 4 \pi$ (where $\Omega$ is the solid angle subtended by the absorber as "seen" from the central quasar). The value of $Q$ is not known, but it is not likely to exceed the detection frequency of AALs among radio-loud quasars ${ }^{8}$, e.g. $\sim 30 \%$ (G. Richards, private communication). If we let $Q_{0.1}$ represent the covering factor relative to $Q=0.1$, the total mass is given by

$$
\begin{gathered}
M \approx 2 \times 10^{9} Q_{0.1}\left(\frac{\mu_{H}}{1.4}\right)\left(\frac{N_{H}}{2 \times 10^{20} \mathrm{~cm}^{-2}}\right) \\
\times\left(\frac{R}{28 \mathrm{kpc}}\right)^{2} \mathrm{M}_{\odot}
\end{gathered}
$$

where $\mu_{H}$ is the mean molecular weight per $\mathrm{H}$ particle. The total kinetic energy in the AAL outflow is therefore,

$K \approx 2 \times 10^{58}\left(\frac{M}{3 \times 10^{9} \mathrm{M}_{\odot}}\right)\left(\frac{v}{1000 \mathrm{~km} \mathrm{~s}^{-1}}\right)^{2} \operatorname{ergs}$

For comparison, the much faster $\left(v \sim 10^{4} \mathrm{~km} \mathrm{~s}^{-1}\right)$ but smaller-scale $(R \sim 0.1 \mathrm{pc})$ BAL outflows observed in other quasars are believed to contain total masses (at any instant) of $\sim 1-10 \mathrm{M}_{\odot}$ for $Q_{0.1} \sim 1$ to 3 . Over a quasar's lifetime, say $10^{8}$ yr (Haehnelt, Natarajan \& Rees 1998), a BAL wind could eject a total of $\sim 10^{7}-10^{8} \mathrm{M}_{\odot}$ with $K \approx 10^{58}-10^{59}$ ergs (Hamann \& Brandt 2000).

8) Emission lines from the AAL gas could be quite strong, depending on the actual values of $U$ or $N_{\mathrm{H}}$. For example, we calculated emission line strengths for a photoionized plasma consistent with the Si II AAL region described above, namely, having solar abundances, an incident spectrum as defined in $\S 4.4$, and the following physical paramters: $R \approx 28 \mathrm{kpc}, \log U \approx-2.8$, $N_{\mathrm{H}}=2 \times 10^{20} \mathrm{~cm}^{-2}$, and $n_{H} \approx 300 \mathrm{~cm}^{-3}$. The strongest predicted emission lines within our wavelength coverage for 3C 191 are $\mathrm{Ly} \alpha$ and $\mathrm{Mg}$ II $\lambda 2799$, with rest-frame equivalent widths of $\sim 44 Q_{0.1} \AA$ and $\sim 4 Q_{0.1} \AA$. These results should be compared to the rest-frame equivalent widths of the measured BELs, e.g. $\sim 120 \AA$ in Ly $\alpha$ and $\sim 17 \AA$ in $\mathrm{Mg}$ II (as estimated without the superposed AALs). The predicted emission lines could therefore be present but "hidden" in the measured

\footnotetext{
${ }^{8}$ The detection frequency sets an approximate upper limit on $Q$ because some fraction of the systems counted as AALs will have a different physical origin than the absorber in $3 \mathrm{C}$ $191(\S 1)$.
} 
BELs. Clearly, however, $Q_{0.1} \sim 1$ is close to an upper limit by this test. Another constraint is that $Q_{0.1}>1$ could lead to too much line emission "filling in" the bottoms of the AAL troughs (depending on the global line-of-sight velocity distribution of the emitting gas; see Hamann, Korista \& Morris 1993 and Hamann \& Korista 1996 for similar arguments related to BALs). Future observations at longer wavelengths might provide more stringent upper limits on $Q_{0.1}$. In particular, our calculations predict that the strongest lines in the rest-frame near-UV/visible should be $\mathrm{H} \alpha, \mathrm{H} \beta$, the O II $\lambda 3727$ doublet, and O III $\lambda 5007$, with rest equivalent widths of $\sim 55 Q_{0.1} \AA, \sim 11 Q_{0.1} \AA$, $\sim 22 Q_{0.1} \AA$, and $\sim 88 Q_{0.1} \AA$, respectively.

\subsection{Toward a Physical Model}

Figure 6 shows a highly schematic model of the AAL region, wherein pockets of dense neutral gas are surrounded by a diffuse, spatially distributed medium of generally higher ionization. The diffuse clouds contain most of the total column density $(\S 4.5)$. Their greater size and/or greater numbers lead to more complete coverage in both velocity and projected area (for example, in the C IV doublet), compared to the lower-ionization gas (e.g. Mg I $\lambda 2853$ ).

Note that the extended regions cannot have exclusively high ionization levels because the measured line-of-sight coverage fractions do not correlate simply with ionization. For example, low ionization lines can have either high (e.g. C II $\lambda 1334$, Si II $\lambda 1260)$ or low (Fe II) coverage fractions $(\S 4.2)$. The amount of coverage in both space and velocity must depend at least partly on the line's oscillator strength. In other words, the coverage fraction scales with the line optical depth. Stronger (more optically thick) lines have greater contributions from the diffuse extended gas, resulting in greater coverage, whereas weak lines sample mainly the higher column density material in more compact regions.

A major concern with any cloud model is the cloud survival. The lifetime of a cloud without pressure confinement is of order the sound-crossing time. For a nominal temperature of $10^{4} \mathrm{~K}$ and a maximum cloud size of $\Delta R \lesssim 0.2 \mathrm{pc}$ (Eqn. 11), the cloud survival time, $\lesssim 2 \times 10^{4}$ yr, is much less than the characteristic flow time, $t \approx 3 \times 10^{7}$ yr (Eqn 10). Therefore pressure confinement ap- pears necessary. The problem of understanding this confinement has plagued cloud models of both the BAL and BEL regions of AGNs. Possible solutions include external pressure from a magnetic field or a surrounding hot, low-density (and transparent) plasma (Weymann, Turnshek \& Christiansen 1985, Arav, Li \& Begelman 1994, Emmering, Blandford \& Shlosman 1992, DeKool 1997, Feldmeier et al. 1997).

The key remaining question is, what provides the source of material and kinetic energy for the AAL outflow? The flow time $\left(\sim 3 \times 10^{7}\right.$ $\mathrm{yr})$ is comparable to predicted quasar lifetimes (Terlevich \& Boyle 1993, Haehnelt et al. 1998). It therefore seems likely that the AAL gas originated much nearer the quasar, perhaps coincident with the onset of quasar activity.

We have already noted ( $\$ 5.1$, item 7$)$ that the kinetic energy in this AAL region is comparable to the typical energy in BAL winds (integrated over a quasar's lifetime). Therefore, quasars are capable of driving winds with this total energy. In addition, most current models of BAL winds have them preferentially located near the plane of the accretion disk (Murray \& Chiang 1995, Emmering et al. 1992, Wills, Brandt \& Laor 1999 and references therein). This geometry is reminiscent of the equatorial structure (tentatively) inferred for AAL regions in radio-loud quasars $(\S 1)$.

However, it is unlikely that the AAL outflow in 3C 191 is simply an extended remnant of a BAL wind because 1) the terminal velocity of a BAL wind should be of order $10^{4} \mathrm{~km} \mathrm{~s}^{-1}$ instead of 1000 $\mathrm{km} \mathrm{s}^{-1}$, and 2) the total mass in AAL gas is at least an order of magnitude larger than expected for BAL winds (item 7 in $\S 5.1$ ). To produce the observed AALs, a high-velocity BAL-like wind would have to be decelerated by interaction with ambient galactic material and then, probably, entrain some of that material (to add mass) along the way.

An alternative possibility is that the AALs form in gas that was expelled by stellar processes, e.g. in a galactic "superwind" as observed in lowredshift starburst galaxies (Heckman, Armus \& Miley 1990, Heckman et al. 2000). The sizes, masses, velocities, etc. inferred for superwinds in luminous starbursts are consistent with our estimates of these quantities in 3C 191. The superwinds also contain cool dense clouds (giving 
rise to $\mathrm{Na} \mathrm{I}$ absorption lines) embedded in a hot $\left(\sim 10^{7} \mathrm{~K}\right)$ X-ray emitting plasma (see also Heckman et al. 1996 and references therein). If a superwind model does apply to 3C 191, the characteristic flow time of the AAL gas $\left(\sim 3 \times 10^{7}\right.$ yr) might represent the time elapsed since the starburst episode.

No matter what scenario accounts for the AALs in 3C 191, it is important to keep in mind that AALs in different objects can probe very different physical phenomena. For example, AALs in other quasars often have higher blueshifted velocities than those in 3C 191. Some narrow absorption lines have blueshifts above the arbitrary 5000 $\mathrm{km} \mathrm{s}^{-1}$ AAL threshold, even though there is strong evidence for their being intrinsic to the quasar environments (Hamann et al. 1997a and 1997b, Barlow \& Sargent 1997, Barlow, Hamann \& Sargent 1997, Richards 2000). A galactic superwind certainly cannot explain these highvelocity absorbers. Most AAL systems also do not have low-ionization lines like 3C 191 (e.g. Junkkarinen, Hewitt \& Burbidge 1991, Hamann 1997). It is possible that low ionization AALs, which allow us to locate the absorber via $\mathrm{Si} \mathrm{II}^{*}$, select in favor of large absorber-quasar distances. In particular, all of the known AAL systems with these excited-state lines have distances $\gtrsim 10 \mathrm{kpc}$ (e.g. Barlow et al. 1997, Tripp, Lu \& Savage 1996, Morris et al. 1986, Sargent, Boksenberg \& Young 1982). Other AALs are known to form much closer to the quasars, possibly within a few pc in outflows similar to the BALs (Hamann et al. 1997a and 1997b, Barlow \& Sargent 1997, Barlow et al. 1997).

Given this diversity, it is interesting to note that 3C 191 does not follow the trend identified by Brandt et al. (2000) for small X-ray to UV continuum flux ratios accompanying strong C IV absorption equivalent widths (§4.5). That correlation nominally points to a relationship between the strength of the AALs and the strength of continuous (bound-free) absorption in X-rays. BAL quasars are at one extreme in this relationship having both strong UV lines and strong absorption in X-rays (see also Green \& Mathur 1996, Gallagher et al. 1999). 3C 191 might contain a different class of absorber (e.g. much farther from the active nucleus) than the majority of sources discussed by Brandt et al. (2000).
We are grateful to Tom Bida and Bob Goodrich for their help with the Keck observations, and Tony Misch for assistance at the Lick Observatory. We thank Gary Ferland for his continued support and distribution of the CLOUDY software, and, in particular, for providing the $\mathrm{Fe}^{+}$level population results discussed in $\S 4.6$. The work of $\mathrm{FH}$ was funded in part by a NASA grant, NAG 5-3234, and an NSF Career Award, AST-9984040. CBF acknowledges the support of NSF grant AST 9803072 
TABLE 1

AAL Measurements

\begin{tabular}{|c|c|c|c|c|c|c|c|c|c|}
\hline \multirow[b]{2}{*}{ Line } & \multicolumn{4}{|c|}{$\longrightarrow \mathrm{W}_{\lambda}(\AA) \longrightarrow$} & \multicolumn{5}{|c|}{$\longrightarrow \log N\left(\mathrm{~cm}^{-2}\right)$} \\
\hline & $\Delta v_{1}$ & $\Delta v_{2}$ & $\Delta v_{3}$ & Total & $\Delta v_{1}$ & $\Delta v_{2}$ & $\Delta v_{3}$ & Total & $\operatorname{Tot}\left(C_{f}<1\right)^{\mathrm{a}}$ \\
\hline Si III 1206 & 0.04 & 1.46 & 1.19 & 2.70 & 12.5 & 14.5 & 14.2 & 14.6 & - \\
\hline $\operatorname{Ly} \alpha$ & 0.69 & 1.29 & 1.28 & 3.26 & 14.4 & 14.9 & 14.7 & 15.2 & - \\
\hline N V average & 0.36 & 0.96 & 0.38 & 1.70 & 14.5 & 15.4 & 14.8 & 15.5 & - \\
\hline Si II 1260 & 0.12 & 1.04 & 0.80 & 1.95 & 13.0 & 14.3 & 14.0 & 14.5 & - \\
\hline Si II* 1265 & - & 0.85 & 0.14 & 0.97 & - & 14.1 & 13.1 & 14.2 & - \\
\hline Si II 1304 & - & 0.44 & 0.19 & 0.63 & - & 14.7 & 14.3 & 14.8 & - \\
\hline Si II* 1309 & - & 0.13 & 0.04 & 0.17 & - & 14.2 & 13.6 & 14.3 & - \\
\hline $\mathrm{C}$ II+C II* & - & - & - & 2.49 & - & - & - & 15.2 & - \\
\hline Si IV 1394 & 0.18 & 1.29 & 1.17 & 2.63 & 13.4 & 14.6 & 14.4 & 14.8 & - \\
\hline Si IV 1403 & 0.14 & 1.22 & 0.97 & 2.32 & 13.5 & 14.8 & 14.6 & 15.0 & - \\
\hline Si II 1527 & 0.03 & 0.65 & 0.32 & 1.00 & 13.0 & 14.5 & 14.1 & 14.7 & 15.1 \\
\hline Si II* 1533 & - & 0.38 & 0.08 & 0.46 & - & 14.2 & 13.5 & 14.3 & 14.5 \\
\hline C IV 1548 & 0.71 & 1.66 & - & - & 14.6 & 15.1 & - & - & - \\
\hline C IV 1551 & - & 1.66 & 1.39 & - & - & 15.4 & 15.2 & - & - \\
\hline C IV total & - & - & - & 6.12 & - & - & - & 15.7 & - \\
\hline Fe II 1608 & - & 0.04: & - & 0.04: & - & 13.4: & - & 13.4: & 13.5: \\
\hline Al II 1671 & - & 0.72 & 0.37 & 1.09 & - & 13.4 & 13.0 & 13.5 & 13.9 \\
\hline Si II 1808 & - & 0.05: & - & $0.05:$ & - & 14.9: & - & 14.9: & 15.0: \\
\hline Al III 1855 & - & 0.77 & 0.25 & 1.02 & - & 13.8 & 13.2 & 13.9 & 14.4 \\
\hline Al III 1863 & - & 0.60 & 0.19 & 0.78 & - & 14.0 & 13.4 & 14.1 & 14.3 \\
\hline Fe II 2344 & - & 0.30 & 0.08 & 0.38 & - & 13.7 & 13.2 & 13.9 & 14.0 \\
\hline Fe II 2374 & - & 0.08: & - & 0.08: & - & 13.5: & - & 13.5: & 13.7: \\
\hline Fe II 2383 & - & 0.63 & 0.30 & 0.92 & - & 13.6 & 13.3 & 13.8 & 14.0 \\
\hline Fe II 2587 & - & 0.30: & - & 0.30: & - & 14.0: & - & 14.0: & 14.2: \\
\hline Fe II 2600 & - & 0.59 & 0.23 & 0.82 & - & 13.7 & 13.2 & 13.9 & 14.1 \\
\hline Mg II 2796 & 0.07 & 2.10 & 1.39 & 3.55 & 12.3 & 14.0 & 13.7 & 14.1 & 14.4 \\
\hline Mg II 2804 & - & 1.69 & 1.00 & 2.70 & - & 14.1 & 13.8 & 14.3 & 14.4 \\
\hline Mg I 2853 & - & 0.55 & 0.24 & 0.79 & - & 12.7 & 12.3 & 12.9 & 13.1 \\
\hline
\end{tabular}

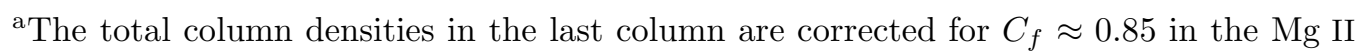
doublet and $C_{f} \approx 0.70$ in other lines. Lines without entries in this column are poorly measured or appear very optically thick $(\S 4.3)$. 


\section{References}

Akujor, C.E., Lüdke, E., Browne, I.W.A., Leahy, J.P., Garrington, S.T., Jackson, N., \& Thomasson, P. 1994, A\&AS, 105, 247

Anderson, S.F., Weymann, R.J., Foltz, C.B. \& Chaffee, F.H. 1987, AJ, 94, 278

Arav, N., Li, Z.-Y., \& Begelman, M.C. 1994, ApJ, 432,62

Arav, N., Brotherton, M.S., Becker, R.H., Gregg, M.D., White, R.L., Price, T., \& Hack, W. 2000, preprint (astro-ph/0008259)

Bahcall, J.N., Sargent, W.L.W., \& Schmidt, M. 1967, ApJ, 149, L11

Bahcall, J.N., \& Wolf, R.A. 1968, ApJ, 152, 701

Barlow, T. A., Hamann, F., \& Sargent, W. L. W. 1997, in Mass Ejection From AGN, eds. R. Weymann, I. Shlosman, and N. Arav, ASP Conf. Series, 128, 13

Barlow, T. A., \& Sargent, W. L. W. 1997, AJ, 113, 136

Barthel, P., Tytler, D.R., \& Vestergaard, M. 1997, in Mass Ejection From AGN, eds. R. Weymann, I. Shlosman, and N. Arav, ASP Conf. Series, 128, 48

Brandt, W.N., Laor, A., \& Wills, B.J. 2000, ApJ, 528, 637

Brotherton, M.S., Wills, B.J., Dey, A., van Bruegel, W., \& Antonucci, R. 1998, ApJ, 501, 110

Burbidge, E.M., Lynds, C.R., \& Burbidge, G.R. 1966, ApJ, 144, 447

Cardelli, J.A., Clayton, G.C., \& Mathis, J.S. 1989, ApJ, 345, 245

Carroll, S.M., Press, W.H., \& Turner, E.L. 1992, ARA\&A, 30, 499

Crenshaw, D.M., Kraemer, S.B., Boggess, A., Stephen, P., Mushotzky, R.F., \& Wu, C.-C. 1999, ApJ, 516, 750

de Kool, M. 1997, in Mass Ejection From AGN, eds. R. Weymann, I. Shlosman, and N. Arav, ASP Conf. Series, 128, 233

Emmering, R.T., Blandford, R.D., \& Shlosman, I. 1992, ApJ, 385, 460

Fall, S.M., \& Pei, Y.C. 1989, ApJ, 337, 7

Feldmeier, A., Norman, C., Pauldrach, A. Owocki, S., Puls, J., \& Kaper, L. 1997, in Mass Ejection From AGN, eds. R. Weymann, I. Shlosman, and N. Arav, ASP Conf. Series, 128, 258

Ferland, G.J., Korista, K.T., Verner, D.A., Ferguson, J.W., Kingdon, J.B., \& Verner, E.M.
1998, PASP, 110, 761

Foltz, C.B, Weymann, R.J., Peterson, B.M., Sun, L., Malkan, M.A., \& Chaffee, F.H. 1986, ApJ, 307,504

Galavis, M.E., Mendoza, C. \& Zeippen, C.J. 1998, A\&AS, 131, 499

Gallagher, S.C., Brandt, W.N., Sambruna, R.M., Mathur, S., \& Yamasaki, N. 1999, ApJ, 519, 549

Green, P., \& Mathur, S. 1996, ApJ, 462, 637

Grevesse, N., \& Anders, E. 1989, in Cosmic Abundances of Matter, ed. C.I. Waddington, AIP Conf. Proc., 183, 1

Haehnelt, M.G., Natarajan, P., \& Rees, M.J. 1998, MNRAS, 300, 817

Halpern, J.P., Eracleous, M., Filippenko, A.V., \& Chen, K. 1996, ApJ, 464, 704

Hamann, F. 1997, ApJS, 109, 279

Hamann, F., Barlow, T. A., Junkkarinen, V. T., \& Burbidge, E. M. 1997a, ApJ, 478, 80

Hamann, F., Barlow, T. A., \& Junkkarinen, V. T. 1997b, ApJ, 478, 87

Hamann, F., \& Brandt, W.N. 2000, PASP, review in prep.

Hamann, F., \& Ferland, G.J. 1999, ARA\&A, 37, 487

Hamann, F., \& Korista, K.T. 1996, ApJ, 464, 158

Hamann, F., Korista, K.T., \& Morris, S.L. 1993, ApJ, 415, 541

Hamann, F., Netzer, H., \& Shields, J.C. 2000, ApJ, 536, 101

Heckman, T.M., Armus, L., \& Miley, G.K. 1990, ApJS, 74, 833

Heckman, T.M., Dahlem, M., Stephan, Eales, S.A., Fabbiano, G., \& Weaver, K. 1996, ApJ, 457,616

Heckman, T.M., Lehnert, M.D., Strickland, D.K., \& Armus, L. 2000, ApJ, in press

Hewitt, D. \& Burbidge, G. 1993, ApJS, 87, 451

Junkkarinen, V., Hewitt, D. \& Burbidge, G. 1991, ApJS, 77, 203

Kaspi, S., Smith, P.S., Netzer, H., Maoz, D., Jannuzi, B.T., \& Giveon, U. 2000, ApJ, 533, 631

Laor, A., Fiore, F., Elvis, M., Wilkes, B. J., \& McDowell, J. C. 1997, ApJ, 477, 93

Marziani, P., Sulentic, J.W., Dultzin-Hacyan, D., Calvani, M., \& Moles, M. 1996, ApJS, 104, 37

Mendoza, C. 1983, in Planetary Nebulae, ed. D.R. Flower, (Dordrecht: Reidel), 143 
Meurer, G.R., Heckman, T.M., \& Calzetti, D. 1999, ApJ, 521, 64

Morris, S.L., Weymann, R.J., Foltz, C.B., Turnshek, D.A., Shectman, S., Price, C., \& Boroson, T.A. 1986, ApJ, 310, 40

Morrison, R., \& McCammon, D. 1983, ApJ, 270, 119

Murray, N., \& Chiang, J. 1995, ApJ, 454, L105

Murray, N., Chiang, J., Grossman, S. A., \& Voit, G. M. 1995, ApJ, 451, 498

Netzer, H. 1992, AIP Conf. Proc. 254, Testing the AGN Paradigm, ed. S.S. Holt, S.G. Neff, \& C.M. Urry (New York:AIP), 146

O'Brien, P.T., Gondhalekar, P.M., \& Wilson, R. 1988, MNRAS, 233, 801

Osterbrock, D.E. 1989, Astrophysics of Gaseuos Nebulae and Active Galactic Nuclei, Mill Valley, Univ. Sci. Press

Peterson, B.M. 1993, PASP, 105, 207

Petitjean, P., Rauch, M., \& Carswell, R. F. 1994, A\&A, 291, 29

Petitjean, P. \& Srianand, R. 1999, å, 345, 73

Rauch, M. 1998, ARA\&A, 36, 267

Richards, G.T. 2000, ApJS, submitted

Richards, G.T., Laurent-Muehleisen, R.H. Becker, \& York, D.G. 2000, ApJ, submitted

Sargent, W.L.W., Boksenberg, A. \& Young, P. 1982, ApJ, 252, 54

Savage, B.D., \& Sembach, K.R. 1991, ApJ, 379, 245

Stockton, A.N., \& Lynds, C.R. 1966, ApJ, 144, 451

Terlevich, R.J., \& Boyle, B.J. 1993, MNRAS, 262, 491

Tripp, T.M., Lu, L., \& Savage, B.D. 1996, ApJS, 102, 239

Turnshek, D. A. 1988, in QSO Absorption Lines: Probing the Universe, eds. J. C. Blades, D. A. Turnshek, \& C. A. Norman (Cambridge: Cambridge Univ. Press), 17

Tytler, D., \& Fan, X.-M. 1992, ApJS, 79, 1

Verner, D., Verner, E.M., \& Ferland, G.J. 1996, Atomic Data and Nucl. Data Tables, 64, 1

Vogt, S.S., et al. 1994, Proc. SPIE, 2198, 362

Wampler, E.J., Chigai, N.N., \& Petitjean, P. 1995, ApJ, 443, 586

Weymann, R.J., Morris, S.L., Foltz, C.B., \& Chaffee, F.H. 1991, ApJ, 373, 465

Weymann, R.J., Turnshek, D.A., \& Christiansen, W.A. 1985, in Astrophysics of Active Galactic
Nuclei and Quasistellar Objects, ed. J. Miller (Oxford:Oxford Univ. Press), 333

Weymann, R.J., Williams, R.E., Peterson, B.M., \& Turnshek, D.A. 1979, ApJ, 234, 33

Wilkes, B.J., Tananbaum, H., Worrall, D.M., Avni, Y., Oey, M.S., \& Flanagan, J. 1994, ApJS, 92, 53

Williams, R.E., Strittmatter, P.A., Carswell, R.F., \& Craine, E.R. 1975, ApJ, 202, 296

Wills, B.J., Thompson, K.L., Han, M., Netzer, H., Wills, D. et al. 1995, ApJ, 447, 139

Zheng, W., Kriss, G.A., Telfer, R.C., Grimes, J.P., \& Davidson, A. F. 1997, ApJ, 475, 469

\section{FIGURE CAPTIONS}

Figure 1. - Part of the measured spectrum of 3C 191 showing the strong associated absorption lines (labeled above). The flux has units $10^{-15}$ $\operatorname{ergs~} \mathrm{cm}^{-2} \mathrm{~s}^{-1} \AA^{-1}$.

Figures $2 \mathrm{a}$ and $2 \mathrm{~b}$. - AAL profiles on a velocity scale defined by the BEL redshift, $z_{e}=$ 1.956. The lines C II $\lambda 1335$ and $\mathrm{C} \mathrm{II}^{*} \lambda 1336$ are blended togther, as are C IV $\lambda 1548$ and $\lambda 1551$, causing them to appear in each other's panel. The feature labeled "N V average" is an average of the N V doublet $\lambda 1239$ and $\lambda 1243$. The sharp features overlying the Fe II $\lambda 2587$ and $\lambda 2600$ profiles are telluric $\mathrm{O}_{2}$ lines. Dotted vertical lines are drawn to guide the eye.

Figure 3. - Observed profiles of Mg II $\lambda 2804$, $\mathrm{Al}$ III $\lambda 1863$ and Fe II $\lambda 2344$ (bold solid lines in each panel) are compared to predicted profiles in these lines based on $\mathrm{Mg}$ II $\lambda 2796, \mathrm{Al}$ III $\lambda 1855$ and Fe II $\lambda 2383$ (thin lines). The thin curve near the bottom of each panel shows the variance per measured pixel. The vertical dotted lines mark the same velocities as Figure 2. The deeper observed compared to predicted profiles indicate partial coverage of the background light source. See $\S 4.2$.

Figure 4. - Theoretical ionization fractions in photoionized, optically thin $\left(\tau_{L C}<1\right)$ clouds having different ionization parameters, $U$. The curves for different metal ions are labeled near their peak values whenever possible. The curves for Fe II, Al II and Al III are dashed for clarity. 
The H I fraction is shown across the top. See $§ 4.4$.

Figure 5. - Theoretical column density ratios in photoionized, optically thin clouds with solar element abundances and different $U$. The measured column densties (Table 1) compared favorably with these predictions if $-3.4 \lesssim \log U \lesssim$ -2.8 . See $\S 4.7$.

Figure 6. - Schematic representation of the AAL environment, showing pockets of dense neutral gas (filled black circles) surrounded by a less dense and more highly ionized medium (grey circles). The more extended regions produce smoother AAL profiles and more complete spatial coverage of the background light source ( 55.2$)$. 


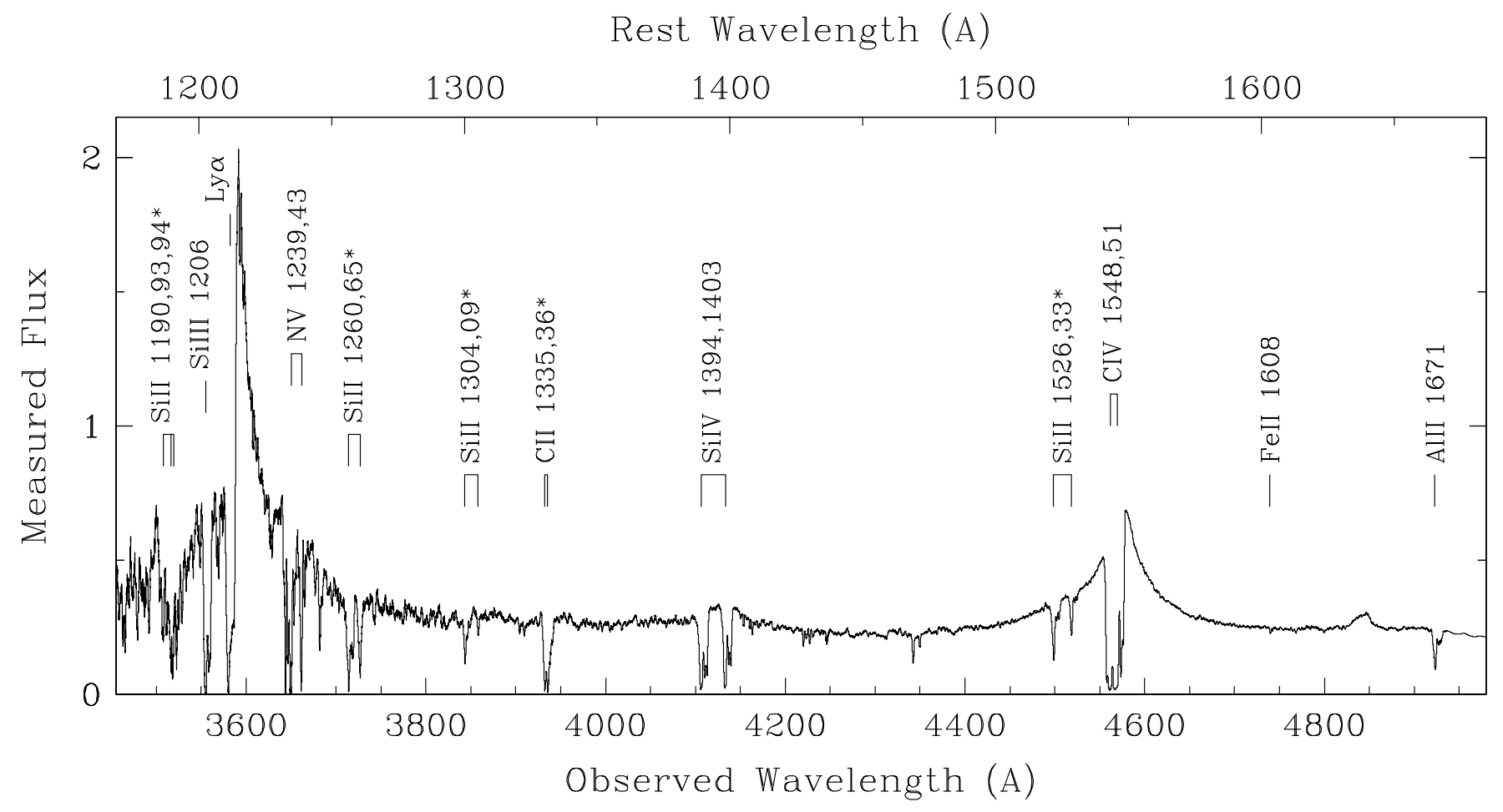




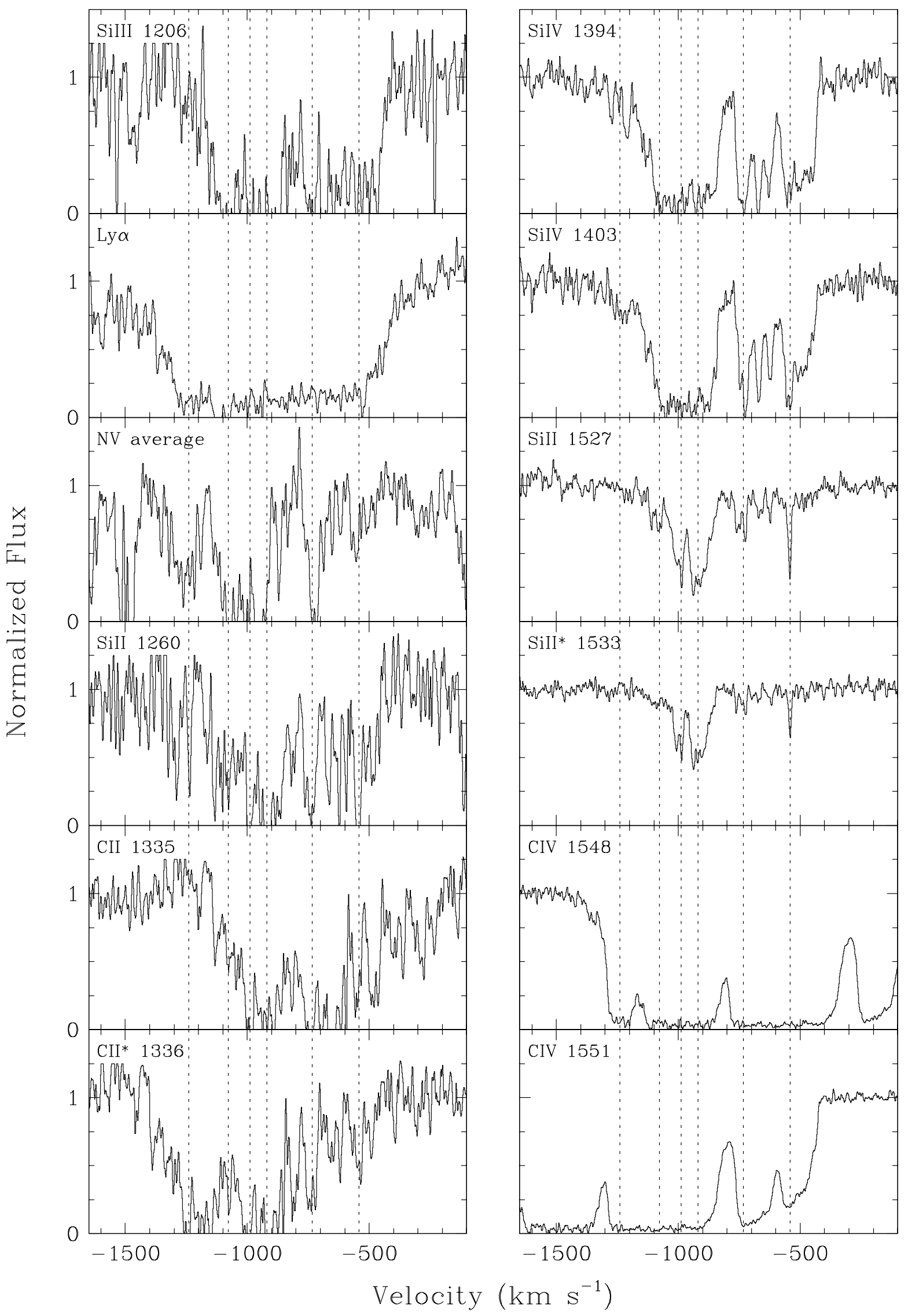




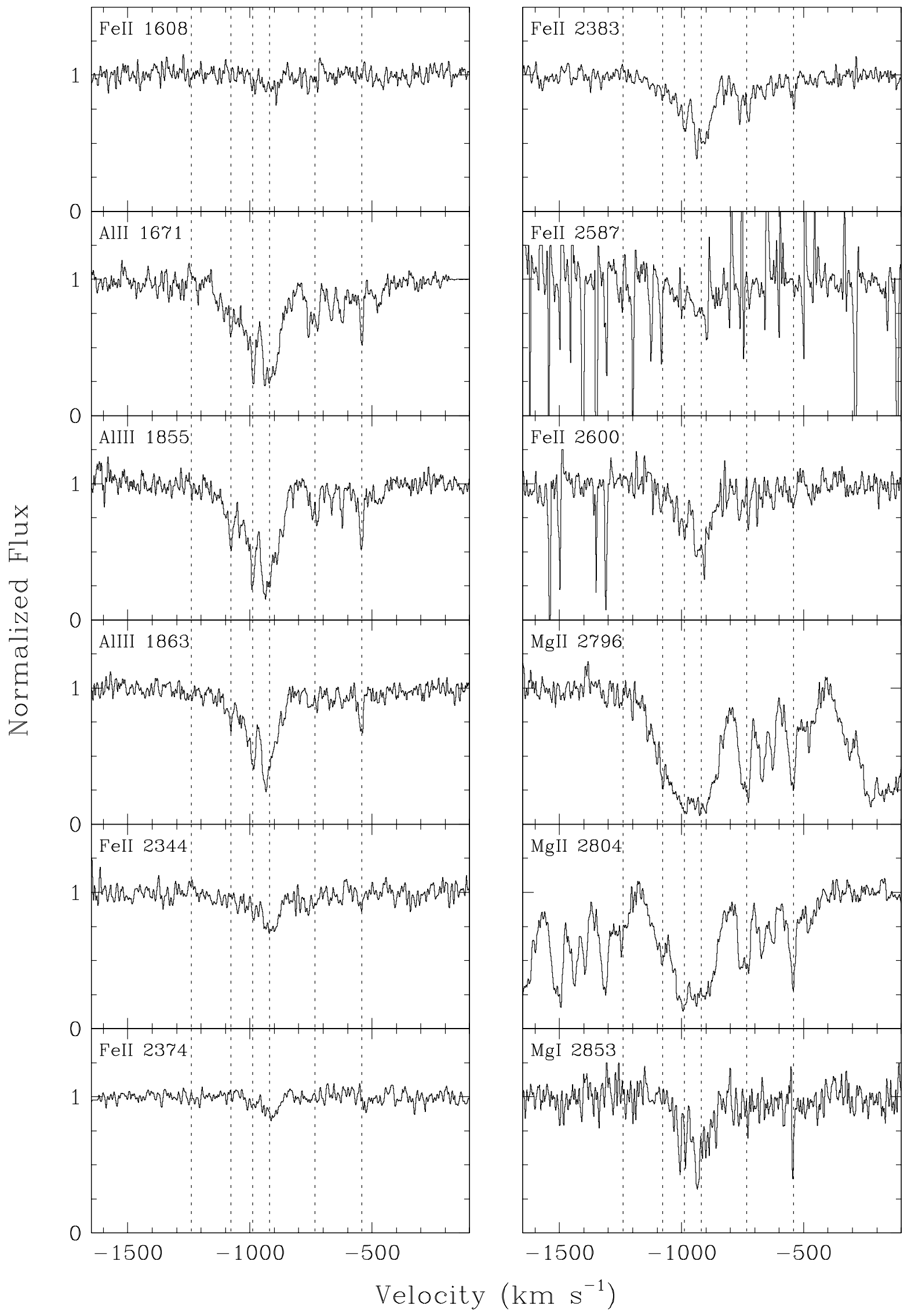




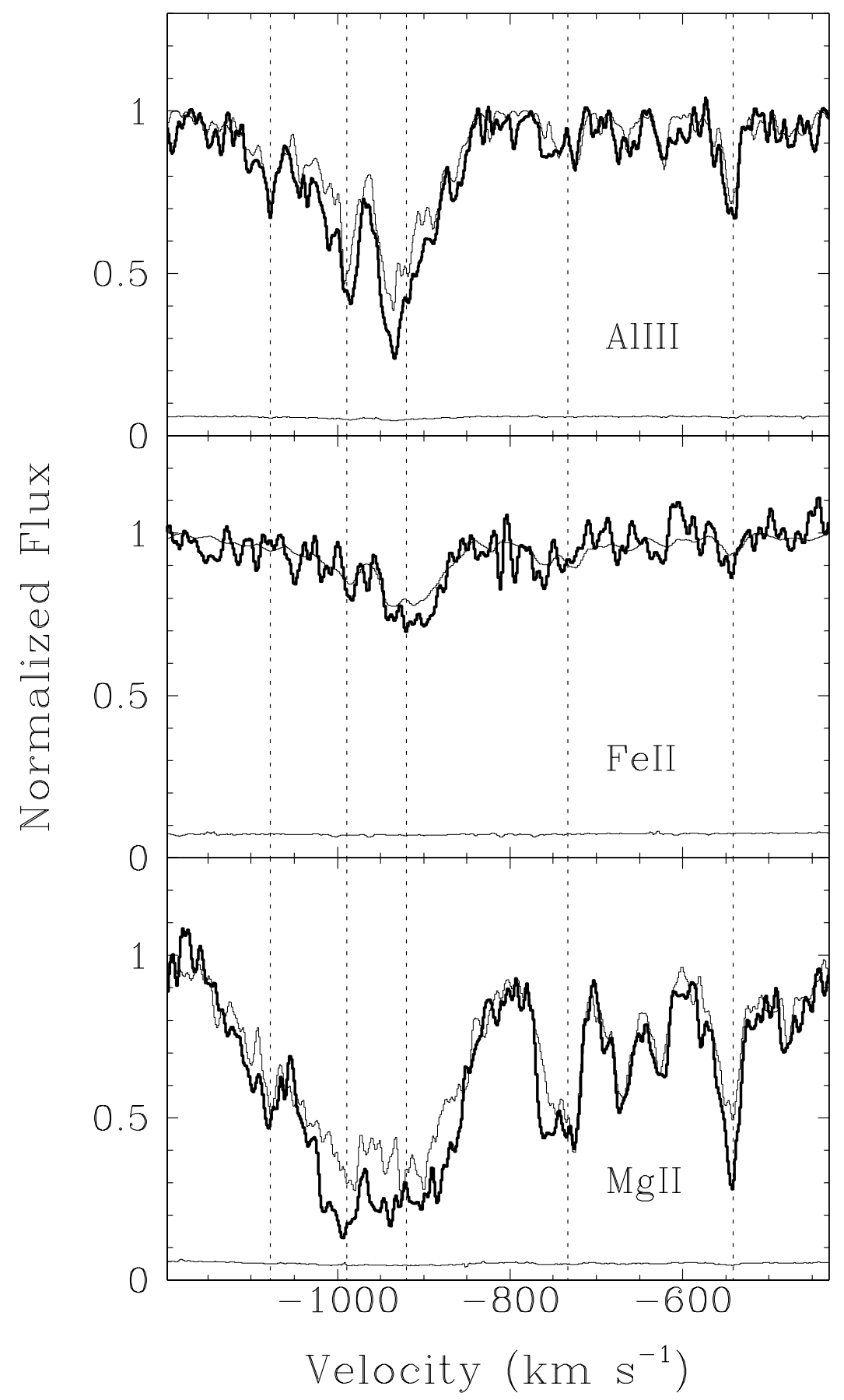




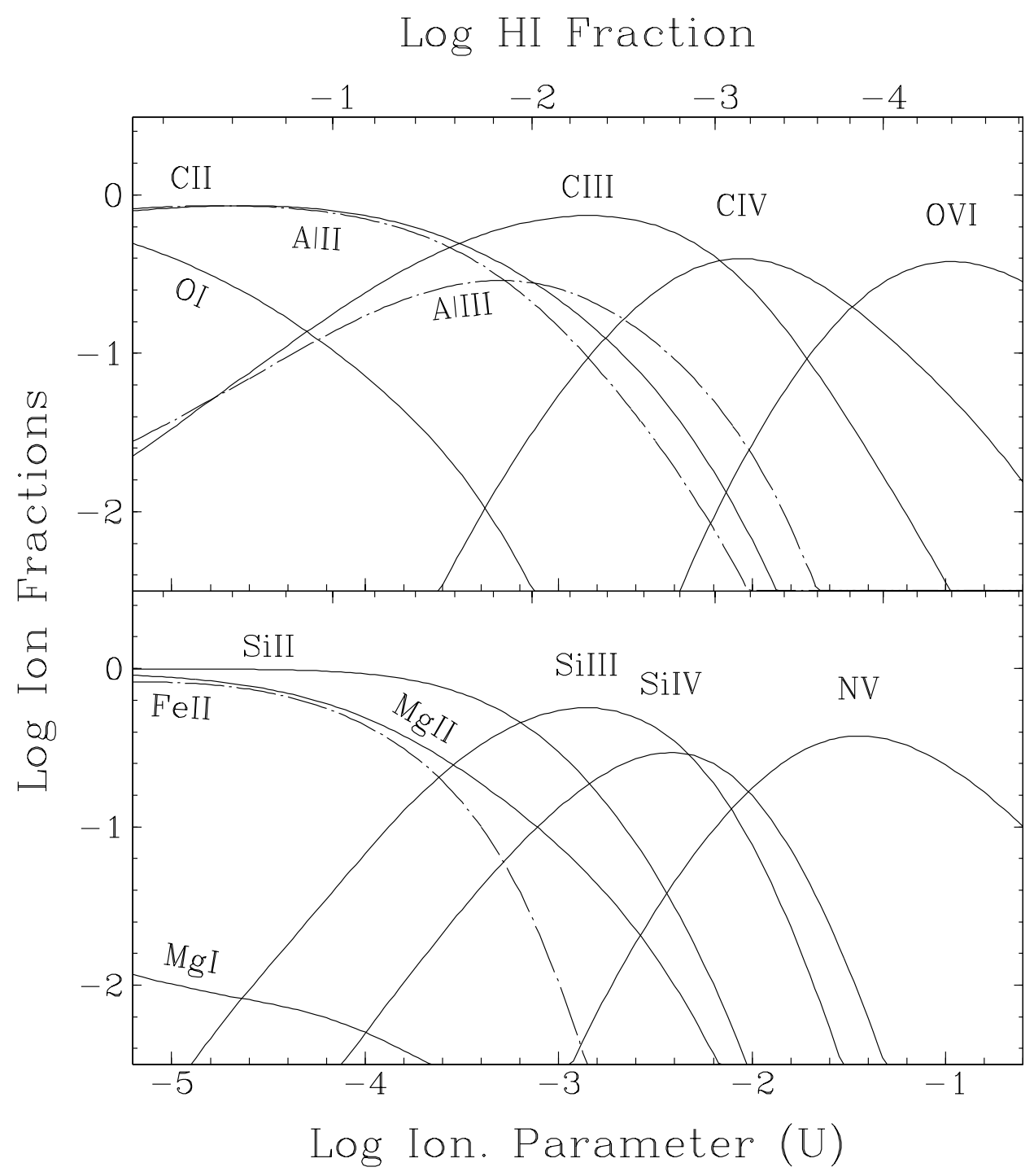




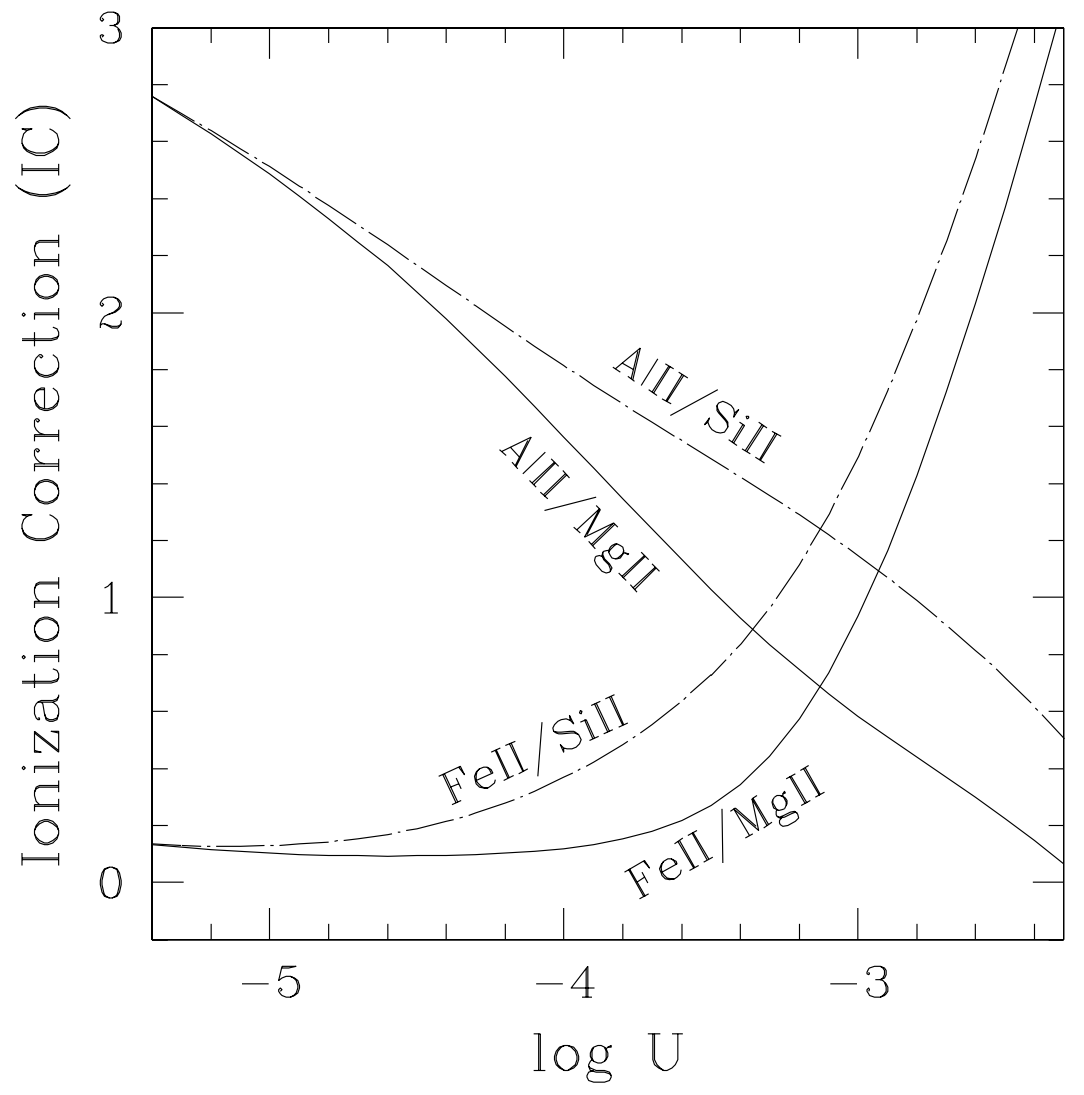




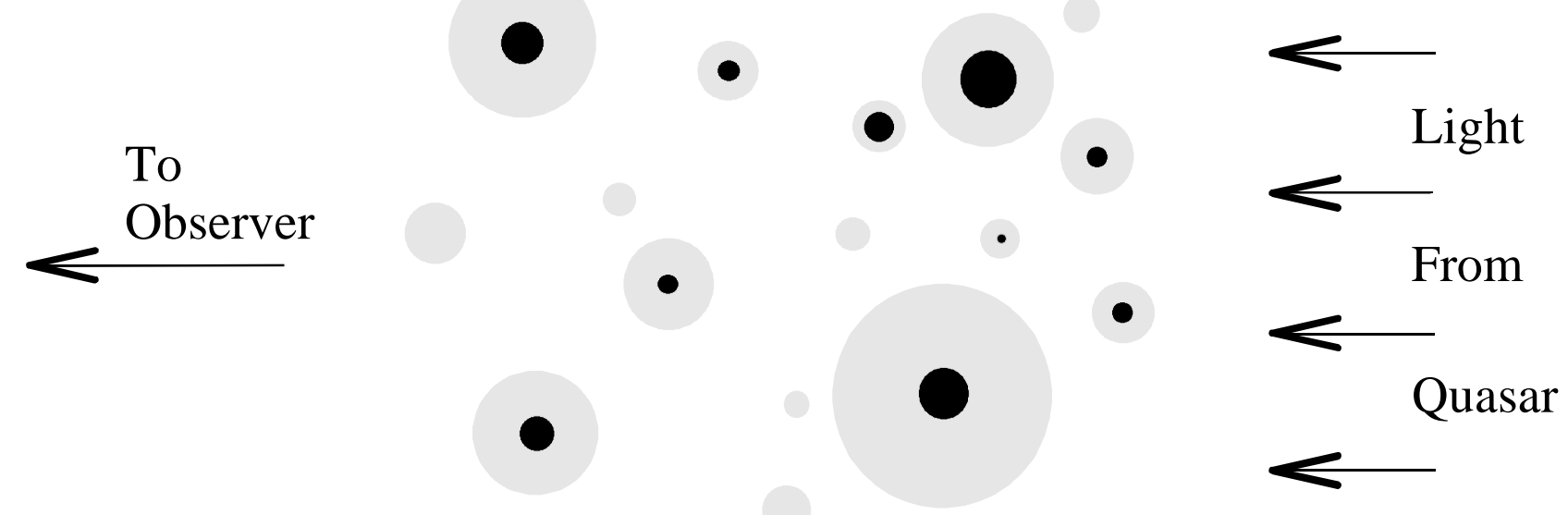

